\title{
Orbital Magnetic Field Effects in Mott Insulators with Strong Spin-Orbit Coupling
}

\author{
Willian M. H. Natori, ${ }^{1}$ Roderich Moessner, ${ }^{2}$ and Johannes Knolle ${ }^{1}$ \\ ${ }^{1}$ Blackett Laboratory, Imperial College London, London SW7 2AZ, United Kingdom \\ ${ }^{2}$ Max-Planck-Institut fur Physik komplexer Systeme, Nöthnitzer Straße 38, 01187 Dresden, Germany
}

\begin{abstract}
We study the effect of a magnetic field on the low energy description of Mott insulators with strong spin-orbit (SO) coupling. In contrast to the standard case of the Hubbard model without SO coupling, we show that Peierls phases can modulate the magnetic exchange at leading order in the interaction. Our mechanism crucially depends on the existence of distinct exchange paths between neighboring magnetic ions enclosing a well-defined area. Thus it will generically be present in any solid state realisation of the Kitaev model and its extensions. We explicitly calculate the variation of the exchange constants of the so-called $J K \Gamma$ model as a function of the magnetic flux. We discuss experimental implications of our findings for various settings of candidate Kitaev spin liquids.
\end{abstract}

Effective low energy descriptions have been crucial for advancing our understanding of correlated quantum phenomena in condensed matter physics as the complexity of microscopic Hamitonians is dramatically reduced. The canonical example is the derivation of the Heisenberg model [1] with exchange constant $J \sim t^{2} / U$ from the half filled single band Hubbard model with kinetic energy $t$ and on-site repulsion $U$ in the Mott insulator (MI) limit [2]. Thouless and later Takahashi realised that charge fluctuations beyond the leading order in the interaction give rise to new higher order ring exchange terms $[2,3]$. These have been extensively discussed in the early context of cuprate high temperature superconductivity [4] where they capture the increased role of charge-, hence, effective quantum-fluctuations when approaching the Mott transition. Moreover, higher order spin exchange terms may stabilise sought after quantum spin liquid (QSL) phases [5-8].

An interesting observation for Hubbard models on nonbipartite lattices was that the application of a magnetic field changes the effective low energy model because charge fluctuations enclosing real space areas lead to higher order spin interactions sensitive to the enclosed magnetic flux [9]. It was shown that the Peierls phases of the orbital magnetic field induce a three-spin scalar chirality term [9] at order $t^{3} / U^{2}$ which is odd under time-reversal symmetry (TRS) and which may again stabilise a QSL phase [10]. In recent years the long search for QSLs [11, 12] has concentrated on MIs in the strong spin-orbit (SO) coupling limit [13-15] following the proposal [16] that they might be described by the Kitaev honeycomb model (KHM) at low energies [17].

Here, we investigate how the low energy description of MI with strong SO coupling is modified in presence of a magnetic field. We discover that in contrast to the Hubbard model, the exchange constants are modified to leading order in the interactions; and that TRS odd terms such as a diamagnetic Zeeman term appear also for the bipartite honeycomb lattice. For most Kitaev transition metal candidates, such as $\alpha-\mathrm{RuCl}_{3}$, the modulation turns out to be small - at most relevant in the pulsed magnetic field regime to study the polarized phase - because the small area between magnetic ions

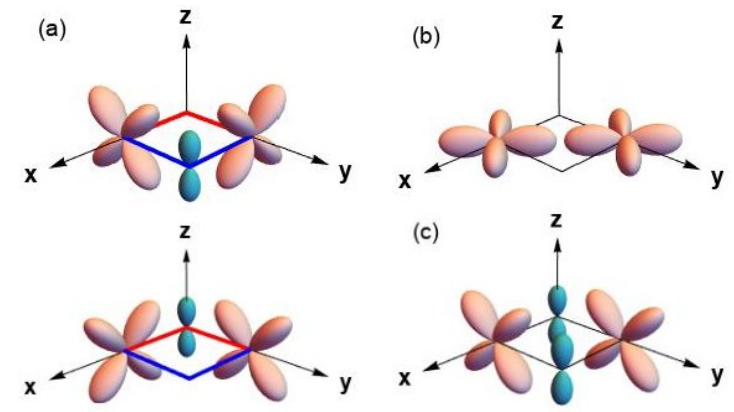

Figure 1: (Color online) Active orbitals on the $x y$-plane for the tight-binding Hamiltonians (a) $\hat{T}_{2}$, (b) $\hat{T}_{3}$, and (c) $\hat{T}_{1}$. The path assigned in blue (red) gives rise to the phase $\phi_{1}$ $\left(\phi_{2}\right)$ discussed in the main text.

leads only to a small value of enclosed flux. However, our basic mechanism will be relevant for the recent Kitaev material proposal in metal-organic frameworks (MOFs) $[18,19]$, where the presence of organic ligands leads to larger distances between the magnetic ions, and hence considerably increased fluxes enclosed by the exchange pathways.

Our paper is organised as follows. First, we provide the necessary background on the physics of magnetic exchange in our class of SO-coupled magnets, specifically introducing the so-called $J K \Gamma$ model. We then include the effect of the magnetic field by applying the Peierls substitution on the hopping integrals of the transfer processes indicated in Fig. 1. Projecting to the low energy manifold we obtain a modified model with couplings dependent on the magnetic flux over areas $\mathcal{A}$ depicted in Fig. 2a. Next, we discuss the resulting modulation of the exchanges. Finally, we assess its relevance for tuning currently available Kitaev materials into QSL phases and for cold atom proposals of the KHM.

Our starting point is the seminal paper Jackeli and Khaliullin, who argued that the KHM emerges at low energies in transition metal compounds with $4 d^{5}$ or $5 d^{5}$ magnetic ions (M) with strong SO coupling [16]. Each transition metal ion is surrounded by six anions (X) forming a perfectly octahedral environment $\mathrm{MX}_{6}$, such that 
the site is described by a single hole occupying one $t_{2 g}$ orbital. Ref. [16] dedicated special attention to the case of compounds with edge-sharing $\mathrm{MX}_{6}$ octahedra, since in this case the M ions can form honeycomb lattices. Symmetry constraints between the orbital shapes and octahedral arrangements motivated the study of superexchange processes driven by the hole transfers occurring along the two distinct paths displayed in figure 1(a). Secondorder perturbation theory is then used to derive effective Kugel-Khomskii models [20, 21] for each of the possible exchange paths, which are then projected to a $j=1 / 2$ Hilbert space defined by the strong SO coupling. The resulting Hamiltonians in each path are quantum compass models [22] that combine the KHM and Heisenberg interactions. With time reversal symmetry the sum of all these compass models cancels the Heisenberg terms and retains only the Kitaev model. This we show is no longer the case in the presence of a magnetic field.

Following Ref. [16] several compounds were synthesised, but they often display long range magnetic order $[14,15]$. In fact, the magnetism of these so-called $K i$ taev Materials is not described by the pure KHM as other hole-transfer processes lead to more complicated effective Hamiltonians such as the Heisenberg-Kitaev (HK) model $[23,24]$ and the $J K \Gamma$ model. Here, we concentrate on the derivation of the $J K \Gamma$ model as a minimal microscopic model of Kitaev materials combining Kitaev, Heisenberg and symmetric off-diagonal exchanges [25, 26].

Studies of the $J K \Gamma$ model $[25,26]$ showed that a QSL would be stable only for materials that are very close to the idealised situation described in Ref.[16]. To suppress the residual magnetism and induce a genuine QSL phase a promising route is to tune parameters of the $J K \Gamma$ model via external fields such as pressure $[27,28]$ or via an applied magnetic field $\mathbf{B}$. The latter route to a QSL is being investigated theoretically [29-36] and experimentally in the candidate material $\alpha-\mathrm{RuCl}_{3}$, which displays a zigzag order in the absence of a magnetic field $\mathbf{B}$ [3739] but an apparently disordered state when subject to $B \sim 14 \mathrm{~T}$ [40-45]. A central question which we address is whether the magnetic field induces additional changes beyond the commonly assumed simple Zeeman term. As the central result we find that a magnetic field modulates the exchange couplings of the $J K \Gamma$ model and induces a diamagnetic Zeeman term in Kitaev materials.

Model Derivation. We begin with the study of the Peierls substitution effects on the original JackeliKhaliullin mechanism. The underlying multi-orbital Hubbard model for Kitaev materials is

$$
H_{\mathrm{Hub}}=\hat{V}+\hat{T}_{2},
$$

where $\hat{V}$ gives the onsite interactions among $t_{2 g}$ holes that depend upon the Coulomb repulsion $U$ and the Hund's coupling $J_{H}[20,21]$ while $\hat{T}_{2}$ is the tight-binding Hamiltonian between the orbitals indicated in Fig. 1(a). $\hat{T}_{2}$ is a bond-dependent Hamiltonian, e.g., only the $|y z\rangle$ and $|z x\rangle$ orbitals are active for bonds on $x y$-planes. The

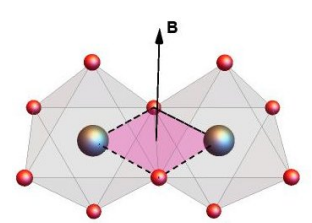

(a)

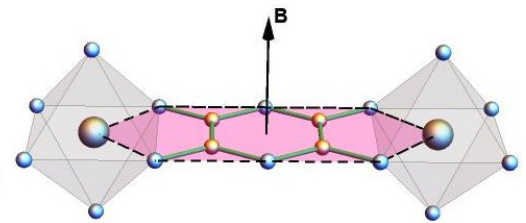

(b)
Figure 2: (Color online) Neighboring octahedra containing the magnetic ions (a) in the originally proposed Kitaev materials and (b) in metal-organic frameworks. The magnetic flux through the $\mathcal{A}$ areas highlighted in magenta gives rise to the Peierls phase in Eq. (5).

hopping integrals $t_{i j}$ in $\hat{T}_{2}$ are modified when the system is subject to a magnetic field B [46]. Within the Peierls substitution, such modification reads

$$
t_{i j} \rightarrow t_{i j} \exp \left(\frac{i e}{\hbar c} \int_{\mathbf{r}_{i}}^{\mathbf{r}_{j}} d \mathbf{r}^{\prime} \cdot \mathbf{A}\left(\mathbf{r}^{\prime}\right)\right)
$$

where $\mathbf{A}$ is the vector potential. In the remainder of the paper, we restrict the discussion to a magnetic field applied in the $\hat{\mathbf{z}}$ direction with $\mathbf{A}=\frac{B}{2}(-y, x, 0)$. We will also focus on minimal models involving two sites $i$ and $j$ connected by a bond $\langle i j\rangle_{z}$ on the $x y$ plane. The relevant terms of $\hat{T}_{2}$ for these two sites are given by

$$
\begin{aligned}
& \hat{T}_{2}^{(1)}=-t_{2} \sum_{\sigma}\left(d_{i, y z, \sigma}^{\dagger} d_{j, z x, \sigma} e^{i \phi_{1}}+d_{j, z x, \sigma}^{\dagger} d_{i, y z, \sigma} e^{-i \phi_{1}}\right), \\
& \hat{T}_{2}^{(2)}=-t_{2} \sum_{\sigma}\left(d_{i, z x, \sigma}^{\dagger} d_{j, y z, \sigma} e^{i \phi_{2}}+d_{j, y z, \sigma}^{\dagger} d_{i, z x, \sigma} e^{-i \phi_{2}}\right),
\end{aligned}
$$

with $t_{2}$ the hopping integral, the superscripts label the two different superexchange paths, and $\phi_{i}$ are the Peierls phases calculated after Eq. (2). The effective model obtained in the strong-coupling approach followed by the projection on the $j=1 / 2$ manifold is the HK model:

$$
\begin{aligned}
H_{\mathrm{Pei},\langle i j\rangle_{z}}^{(1)}= & 4\left[\nu_{1}-\nu_{2}-\left(\nu_{1}+2 \nu_{2}\right) \cos \left(\phi_{1}-\phi_{2}\right)\right] s_{i}^{z} s_{j}^{z} \\
& -2 \nu_{1}\left[1-\cos \left(\phi_{1}-\phi_{2}\right)\right] \mathbf{s}_{i} \cdot \mathbf{s}_{j},
\end{aligned}
$$

in which the energy unity is set at $4 t_{2}^{2} /(9 U)$. The constants $\nu_{1}=\left(3 r_{1}+r_{2}+2 r_{3}\right) / 6$ and $\nu_{2}=\left(r_{1}-r_{2}\right) / 4$ are dependent on the ratios $r_{1}=1 /(1-3 \eta), r_{2}=1 /(1-\eta)$ and $r_{3}=1 /(1+2 \eta)$, where $\eta=J_{H} / U$. The phase difference, Eq. (4), is related to the magnetic field through

$$
\phi_{1}-\phi_{2}=\frac{e}{\hbar c} \oint_{\mathcal{C}} d \mathbf{r}^{\prime} \cdot \mathbf{A}\left(\mathbf{r}^{\prime}\right)=\frac{e}{\hbar c} \int_{\mathcal{A}} d \mathbf{a} \cdot \mathbf{B} \equiv 2 \pi \frac{\Phi}{\Phi_{0}} .
$$

In Eq. (5), $\Phi_{0}$ is the magnetic flux quantum and $\Phi$ is the magnetic flux passing through the area $\mathcal{A}$ enclosed by the superexchange paths, as depicted in Fig. 2. The geometrical symmetry allows us to take $\phi_{2}=-\phi_{1}$ in the chosen gauge, and we use this equality throughout this 
paper. We point out that the magnetic flux piercing the $\mathcal{A}$ areas related to bonds on $z x$ and $y z$ planes is zero, and the Heisenberg interaction is absent in these cases. However, similar HK models will be observed in other planes through the variation of the $\mathbf{B}$ direction.

We are now prepared to discuss the modulation of the $J K \Gamma$ model after applying the Peierls substitution on tight-binding models that include the additional hopping processes indicated in Fig. 1(b) and Fig. 1(c) [18, 25, 26]. Fig. 1(b) illustrates the active orbitals in the tightbinding Hamiltonian $\hat{T}_{3}$. The microscopic details that distinguish $\hat{T}_{3}$ for MOFs and transition metal compounds lead to different ways of applying Peierls substitution. In MOFs, the hopping is mediated by two different paths involving $\sigma$ molecular orbitals [18]. These paths follow closely the ones defined by the Jackeli-Khaliullin mechanism, in such a way that the same phases $\phi_{1,2}$ can be assigned to $\hat{T}_{3}$ under Peierls substitution. By contrast, $\hat{T}_{3}$ in transition metal compounds is related to the direct overlap between the orbitals without any alternative path, which makes the phase defined in Eq. (2) irrelevant. The two cases can be written simultaneously by the Hamiltonian $\hat{T}_{3}=\hat{T}_{3}^{(1)}+\hat{T}_{3}^{(2)}$ given by

$$
\begin{aligned}
& \hat{T}_{3}^{(1)}=-\frac{t_{3}}{2} \sum_{\sigma}\left(d_{i, x y, \sigma}^{\dagger} d_{j, x y, \sigma} e^{i \phi_{a}}+\text { h.c. }\right), \\
& \hat{T}_{3}^{(2)}=-\frac{t_{3}}{2} \sum_{\sigma}\left(d_{i, x y, \sigma}^{\dagger} d_{j, x y, \sigma} e^{i \phi_{b}}+\text { h.c. }\right),
\end{aligned}
$$

where $\phi_{a}=\phi_{1}, \phi_{b}=\phi_{2}$ for MOFs and $\phi_{a}=\phi_{b}=0$ for transition metal compounds. Finally, the active orbitals encompassed by $\hat{T}_{1}$ are illustrated in $1(\mathrm{c})$ and occur when there is a hybridization between the $p$-atomic or $\pi$ molecular orbitals bridging the two sites. The equivalent of the $\mathbf{B}$-induced phase in Eq. (2) should be given by a path integral that considers all classical paths connecting $i$ and $j$, which must be zero by symmetry. The Hamiltonian $\hat{T}_{1}$ then reads

$$
\hat{T}_{1}=-t_{1} \sum_{\sigma}\left(d_{i, y z, \sigma}^{\dagger} d_{j, y z, \sigma}+d_{i, z x, \sigma}^{\dagger} d_{j, z x, \sigma}+\text { h.c. }\right) .
$$

Following the Jackeli-Khaliullin procedure for the tight-binding Hamiltonian $\hat{T}=\hat{T}_{1}+\hat{T}_{2}+\hat{T}_{3}$ yields

$$
\begin{aligned}
H_{\mathrm{Pe} i,\langle i j\rangle_{z}}^{(2)} & =K\left(\phi_{1}\right) s_{i}^{z} s_{j}^{z}+\Gamma\left(\phi_{1}\right)\left(s_{i}^{x} s_{j}^{y}+s_{i}^{y} s_{j}^{x}\right) \\
& +J\left(\phi_{1}\right) \mathbf{s}_{i} \cdot \mathbf{s}_{j}+h\left(\phi_{1}\right)\left(s_{i}^{z}+s_{j}^{z}\right),
\end{aligned}
$$

which is simply the $J K \Gamma$ model with modulated exchange constants and an emergent diamagnetic term. After setting $x_{1} \equiv t_{1} / t_{2}$ and $x_{3} \equiv t_{3} / t_{2}$ we find the analytic ex- pression for the modulated exchanges:

$$
\begin{aligned}
J\left(\phi_{1}\right)= & 4\left[\nu_{1} x_{1}^{2}-\nu_{1} \sin ^{2} \phi_{1}+\frac{\nu_{1}-2 \nu_{2}}{4} x_{3}^{2} \cos ^{2} \phi_{a}\right. \\
& \left.+\left(\nu_{1}+2 \nu_{2}\right) x_{1} x_{3} \cos \left(\phi_{a}\right)\right] \\
K\left(\phi_{1}\right)= & 4\left\{\nu_{2}\left[x_{1}^{2}+x_{3}^{2} \cos ^{2}\left(\phi_{a}\right)-2 x_{1} x_{3} \cos \left(\phi_{a}\right)\right]\right. \\
& \left.\quad+\left[\nu_{1}-\nu_{2}-\cos \left(2 \phi_{1}\right)\left(\nu_{1}+2 \nu_{2}\right)\right]\right\} \\
\Gamma\left(\phi_{1}\right)= & 8 \nu_{2} \cos \phi_{1}\left(x_{1}-x_{3} \cos \phi_{a}\right) \\
h\left(\phi_{1}\right)= & \left(r_{1}+r_{2}\right)\left(x_{1}-x_{3} \cos \phi_{a}\right) \sin \phi_{1} .
\end{aligned}
$$

Equations 9 present the main result of our work - a magnetic field modulation of the exchanges at leading order in the interaction. The emergent diamagnetic term linear in spin operators is somewhat surprising but a direct consequence of the interplay of SO coupling and the broken TRS via the Peierls phases. Note, a magnetic field such as $h\left(\phi_{1}\right)$ has been previously shown to appear in quantum compass model implementations of bosonic optical lattices [47]. There, it is in principle possible to tune the sign of the emergent magnetic field but in contrast here, $h\left(\phi_{1}\right)$ in Eq. (8) always opposes $\mathbf{B}$ and is directly related to the diamagnetism in Kitaev materials.

Finally, we conclude our derivation by noting that a chiral interaction similar to Ref. [10] will also appear in a minimal model including second-neighbor tunneling in the tight-binding Hamiltonian. The hopping on the effective triangular (non-bipartite [9]) lattice will lead to a three-spin interactions including $J^{\prime} \sin \left(\phi_{\triangle}\right) \mathbf{s}_{1} \cdot\left(\mathbf{s}_{2} \times \mathbf{s}_{3}\right)$ with $\phi_{\triangle}$ the flux enclosed by the triangle spanned by the three sublattice sites of a honeycomb plaquette. A recent study of minimal model derivation aided by ab inition techniques [48] showed that Peierls phases indeed induces three-spin interactions on Kitaev materials. However, they were found to be very small to play a significant role for $\alpha-\mathrm{RuCl}_{3}$ and included other interactions besides the chirality term [48]. In the following, we will analyse only the effect of the magnetic field over the two-spin interactions.

Field Modulation of Exchanges. The change of the exchange constants in Eq. (9) as a function of the Peierls phase $\phi_{1}$ and interaction ratio $\eta$ is summarised in Fig. 3. In Fig. 3(a) we use $\eta=0.15$ and electronic parameters $\left(x_{1} \sim 5 / 16, x_{3} \sim-15 / 16\right)$ calculated with ab initio techniques for $\alpha-\mathrm{RuCl}_{3}$ as given by Refs. [15, 26]. A simple figure of merit for how close the model is to the pure Kitaev is the ratio $|K| /(|J|+|\Gamma|)$, in which $K, J$ and $\Gamma$ are given by Eq. (9). For parameters relevant for $\alpha-\mathrm{RuCl}_{3}$, this ratio is maximal when $\phi_{1}=\pi / 2$ and achieves $\approx 2.1$.

Fig. 3(b) provides the analysis of exchange constants with the parameters estimated in Ref. [18] $\left(x_{1}=0.023\right.$, $\left.x_{3}=-0.196\right)$ for MOFs . As indicated by Fig. 3(c), the model Hamiltonian for MOFs is closer to the KHM at 

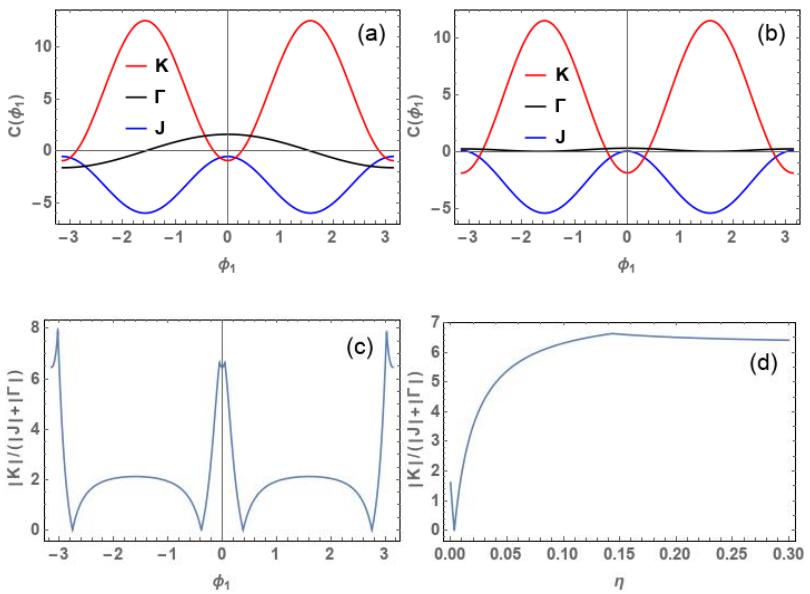

Figure 3: (Color online) Oscillatory behavior of the exchange constants $J\left(\phi_{1}\right), K\left(\phi_{1}\right)$ and $\Gamma\left(\phi_{1}\right)$ with $\eta=0.15$ using (a) the electronic parameters given by Refs. [15, 26] for $\alpha-\mathrm{RuCl}_{3}$ and (b) the electronic parameters for MOFs given by Ref. [18]. Figures (c) and (d) shows the value of $|K| /(|J|+|\Gamma|)$ in MOFs for a fixed value $\eta=0.15$ and a fixed value $\phi_{1}=0.05$, respectively.

$\phi_{1}=0$ with $|K| /(|J|+|\Gamma|) \approx 6.5$. The behaviour of the exchange couplings in the neighbourhood of this point is discussed in the next section. Fig. 3(d) shows that the maximum proximity to the KHM is achieved at $\eta \approx 0.15$ for a physically achievable case $\phi_{1}=0.05$ as discussed in the next section, which is consistent with the Hund's coupling estimation for ruthenium [26].

Experimental Implications. Let us now discuss the relevance of our results for Kitaev candidate materials. We start with the experiments that indicate a spin-liquid behaviour of $\alpha$ - $\mathrm{RuCl}_{3}$ under a strong magnetic field [4045]. Taking the $\mathrm{Ru}-\mathrm{Cl}$ distance to be approximately $2.4 \AA$ [49] and $B \sim 10 \mathrm{~T}, \mathrm{Eq}$. (5) gives a phase difference of $\phi_{1}-\phi_{2} \sim 10^{-2}-10^{-3}$. Using once again the ab initio parameters provided by Refs. [15, 26] and setting $\eta=0.15$, the exchange constants remain practically constant for this magnetic field: $\left[C\left(\phi_{1}\right)-C(0)\right] / C(0) \sim 10^{-4}-10^{-6}$, in which $C$ is $K, J$ or $\Gamma$ in Eq. (9). For this field strength the energy scale of the diamagnetic term $h\left(\phi_{1}\right)$ is $\approx 10^{-2}-10^{-3} \mathrm{meV}$, which is much lower than the scale of the Zeeman effect $(\sim 1 \mathrm{meV})$ and the exchange couplings $(\sim 1-10 \mathrm{meV})[26]$. Therefore, the effects of the Peierls substititution are too small to play a relevant role in the phase transition observed in $\alpha$ - $\mathrm{RuCl}_{3}$. However, for studies of the high-field behaviour our mechanism becomes relevant for this material. Eq. (9) indicates that $J\left(\phi_{1}\right)$ will be reduced by $4 \%$ under a magnetic field of $B \approx 110 \mathrm{~T}$ while $K\left(\phi_{1}\right)$ and $\Gamma\left(\phi_{1}\right)$ remain practically constant. Although it is not feasible to implement a static magnetic field with this intensity, these values are achievable in pulsed high-magnetic field facilities [50] and will be relevant for studying the high field polarised phase, for example for accurately extracting the values of the
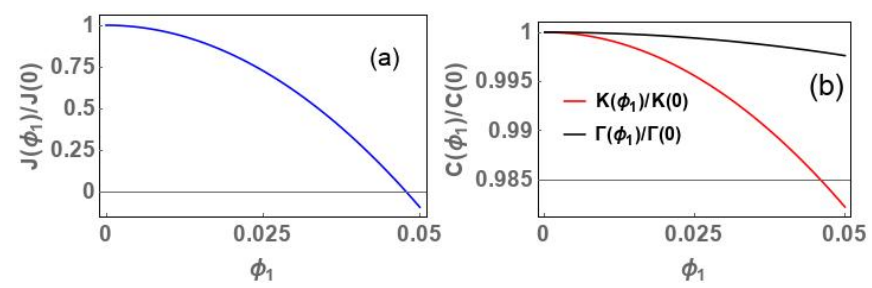

Figure 4: (Color online) Ratios $C\left(\phi_{1}\right) / C(0)$ for Kitaev materials based in metal-organic frameworks for (a) the Heisenberg coupling and (b) the Kitaev and symmetric exchange couplings.

exchange constants.

The larger area $\mathcal{A}$ of MOF systems makes them a much better platform to observe the modulation mechanism. Fig. 4 displays the ratios $C\left(\phi_{1}\right) / C(0)$ of the exchange constants with and without an applied magnetic field. The Heisenberg exchange constant in MOFs also decays much faster than the Kitaev or symmetric exchange couplings, vanishing at $\phi_{1} \approx 0.0479$. This phase can be achieved by applying a (pulsed) magnetic field $B \sim 100 \mathrm{~T}$ over an area $\mathcal{A} \sim 31.5 \AA^{2}$, which is fairly close to the values of $\mathcal{A}$ expected in MOFs [18, 19]. Using $\mathcal{A} \sim 31.5 \AA^{2}$, we also predict a reduction of the Heisenberg coupling by $10 \%$ under an achievable static magnetic field $B \sim 30 \mathrm{~T}$. This reduction could drive an observable phase transition from an ordered phase to a classical spin liquid [51].

Although the phase $\phi_{1}$ is restricted to small values in Kitaev materials, we expect that a much broader range can be controlled in cold-atom implementations of the Kitaev model with similar effects on the exchange couplings. This prediction is based upon results for the minimum spin model in optical lattices with artificial spinorbit coupling. The effect of this spin-orbit coupling was added in terms of a Peierls substitution that led to a quantum compass model with modulated exchange couplings such as in our present study [47]. Similar effects could be observed on KHM realizations in cold atoms $[52,53]$ designed in a way that allows the inclusion of a Peierls phase. For example, the analogous of $\phi_{1}$ in these systems can be controlled with appropriate laser setups $[47,54]$ or using rotating optical lattices $[55,56]$. The minimal model in these systems would display exchange constants that vary similarly to Figs. 3(a) and (b), hence providing tunable experimental settings to study quantum phase transitions.

Conclusion. We have studied the effect of an applied magnetic field on the low energy description of Mott insulators in the strong SO coupling limit. We have concentrated our microscopic calculations on Kitaev materials and uncovered a generic mechanism to modulate effective exchange constant through the application of a strong magnetic field. Since our results are ultimately based only on the existence of more than one exchange path connecting the interacting sites, this mechanism would be at play in all instances of Kitaev materials, 
e.g. also in three-dimensional extensions of the honeycomb lattice $[57,58]$. Similarly, the magnetic field effects on the hopping integrals would lead to an analogous modulation of exchange constants in Kitaev materials based on $f[59,60]$ or $d^{7}$ magnetic species [61, 62]. We propose that the exchange coupling modulation could be observed with current experimental setups in pulsed magnetic field facilities, and possibly with static magnetic fields for MOF systems. The same physical principles could be engineered in optical lattice realizations of the Kitaev model $[52,53]$ and provide a rich platform to study quantum phase transitions.

Finally, it would be desirable to extend our effective low energy models in a field to other correlated quantum materials with sizeable SO couplings, such as parent compounds of iron based superconductors, or effective superlattice structures in the Mott regime where the flux per plaquette is large already for moderate magnetic fields.

Acknowledgements. WMHN and JK acknowledge support from the Royal Society via a Newton International Fellowship. RM acknowledges support from the DFG through SFB 1143 (project-id 247310070) and ct.qmat (EXC 2147, project-id 39085490).

\section{References}

[1] P. W. Anderson, Phys. Rev. 115, 2 (1959).

[2] M. Takahashi, Journal of Physics C: Solid State Physics 10, 1289 (1977).

[3] D. J. Thouless, Proceedings of the Physical Society 86, 893 (1965).

[4] A. H. MacDonald, S. M. Girvin, and D. Yoshioka, Phys. Rev. B 37, 9753 (1988).

[5] G. Misguich, C. Lhuillier, B. Bernu, and C. Waldtmann, Phys. Rev. B 60, 1064 (1999).

[6] W. LiMing, G. Misguich, P. Sindzingre, and C. Lhuillier, Phys. Rev. B 62, 6372 (2000).

[7] J.-Y. P. Delannoy, M. J. P. Gingras, P. C. W. Holdsworth, and A.-M. S. Tremblay, Phys. Rev. B 72, 115114 (2005).

[8] H.-Y. Yang, A. M. Läuchli, F. Mila, and K. P. Schmidt, Phys. Rev. Lett. 105, 267204 (2010).

[9] D. Sen and R. Chitra, Phys. Rev. B 51, 1922 (1995).

[10] O. I. Motrunich, Phys. Rev. B 73, 155115 (2006).

[11] L. Balents, Nature 464, 199 (2010).

[12] J. Knolle and R. Moessner, Annual Review of Condensed Matter Physics 10, 451 (2019), https://doi.org/10.1146/annurev-conmatphys-031218013401 .

[13] J. G. Rau, E. K.-H. Lee, and H.-Y. Kee, Annual Review of Condensed Matter Physics 7, 195 (2016), https://doi.org/10.1146/annurev-conmatphys-031115011319 .

[14] M. Hermanns, I. Kimchi, and J. Knolle, Annual Review of Condensed Matter Physics 9, 17 (2018).

[15] S. M. Winter, A. A. Tsirlin, M. Daghofer, J. van den Brink, Y. Singh, P. Gegenwart, and R. Valentí, Journal of Physics: condensed matter 29, 493002 (2017).

[16] G. Jackeli and G. Khaliullin, Phys. Rev. Lett. 102, 017205 (2009).

[17] A. Kitaev, Ann. Phys. 321, 2 (2006).
[18] M. G. Yamada, H. Fujita, and M. Oshikawa, Phys. Rev. Lett. 119, 057202 (2017).

[19] M. G. Yamada, V. Dwivedi, and M. Hermanns, Physical Review B 96, 155107 (2017).

[20] K. I. Kugel and D. I. Khomskii, Soviet Physics Uspekhi 25, 231 (1982).

[21] G. Khaliullin, Progress of Theoretical Physics Supplement 160, 155 (2005), doi.org/10.1143/PTPS.160.155.

[22] Z. Nussinov and J. van den Brink, Reviews of Modern Physics 87, 1 (2015).

[23] J. Chaloupka, G. Jackeli, and G. Khaliullin, Physical Review Letters 105, 027204 (2010).

[24] J. Chaloupka, G. Jackeli, and G. Khaliullin, Physical Review Letters 110, 097204 (2013).

[25] J. G. Rau, E. K.-H. Lee, and H.-Y. Kee, Physical Review Letters 112, 077204 (2014).

[26] S. M. Winter, Y. Li, H. O. Jeschke, and R. Valentí, Physical Review B 93, 214431 (2016).

[27] L. S. I. Veiga, M. Etter, K. Glazyrin, F. Sun, C. A. Escanhoela, G. Fabbris, J. R. L. Mardegan, P. S. Malavi, Y. Deng, P. P. Stavropoulos, H.-Y. Kee, W. G. Yang, M. van Veenendaal, J. S. Schilling, T. Takayama, H. Takagi, and D. Haskel, Phys. Rev. B 96, 140402 (2017).

[28] R. Yadav, S. Rachel, L. Hozoi, J. van den Brink, and G. Jackeli, Phys. Rev. B 98, 121107 (2018).

[29] Z. Zhu, I. Kimchi, D. N. Sheng, and L. Fu, Phys. Rev. B 97, 241110 (2018).

[30] M. Gohlke, R. Moessner, and F. Pollmann, Phys. Rev. B 98, 014418 (2018).

[31] H.-C. Jiang, C.-Y. Wang, B. Huang, and Y.-M. Lu, arXiv e-prints , arXiv:1809.08247 (2018), arXiv:1809.08247 [cond-mat.str-el] .

[32] L. Zou and Y.-C. He, arXiv e-prints , arXiv:1809.09091 (2018), arXiv:1809.09091 [cond-mat.str-el] .

[33] N. D. Patel and N. Trivedi, arXiv e-prints , arXiv:1812.06105 (2018), arXiv:1812.06105 [condmat.str-el] .

[34] C. Hickey and S. Trebst, Nature Communications 10, 530 (2019).

[35] M.-H. Jiang, S. Liang, W. Chen, Y. Qi, J.-X. Li, and Q.-H. Wang, arXiv e-prints , arXiv:1903.01279 (2019), arXiv:1903.01279 [cond-mat.str-el] .

[36] D. A. S. Kaib, S. M. Winter, and R. Valenti, arXiv e-prints , arXiv:1904.01025 (2019), arXiv:1904.01025 [cond-mat.str-el] .

[37] K. W. Plumb, J. P. Clancy, L. J. Sandilands, V. V. Shankar, Y. F. Hu, K. S. Burch, H.-Y. Kee, and Y.-J. Kim, Physical Review B 90, 041112 (2014).

[38] J. A. Sears, M. Songvilay, K. W. Plumb, J. P. Clancy, Y. Qiu, Y. Zhao, D. Parshall, and Y.-J. Kim, Physical Review B 91, 144420 (2015).

[39] R. D. Johnson, S. C. Williams, A. A. Haghighirad, J. Singleton, V. Zapf, P. Manuel, I. I. Mazin, Y. Li, H. O. Jeschke, R. Valentí, and R. Coldea, Physical Review B 92, 235119 (2015).

[40] J. A. Sears, Y. Zhao, Z. Xu, J. W. Lynn, and Y.-J. Kim, Phys. Rev. B 95, 180411 (2017).

[41] S.-H. Baek, S.-H. Do, K.-Y. Choi, Y. S. Kwon, A. U. B. Wolter, S. Nishimoto, J. van den Brink, and B. Büchner, Phys. Rev. Lett. 119, 037201 (2017).

[42] R. Hentrich, A. U. B. Wolter, X. Zotos, W. Brenig, D. Nowak, A. Isaeva, T. Doert, A. Banerjee, P. LampenKelley, D. G. Mandrus, S. E. Nagler, J. Sears, Y.-J. Kim, B. Büchner, and C. Hess, Phys. Rev. Lett. 120, 117204 
(2018).

[43] A. Banerjee, C. A. Bridges, J.-Q. Yan, A. A. Aczel, L. Li, M. B. Stone, G. E. Granroth, M. D. Lumsden, Y. Yiu, J. Knolle, S. Bhattacharjee, D. L. Kovrizhin, R. Moessner, D. A. Tennant, D. G. Mandrus, and S. E. Nagler, Nature Materials 15, 733 (2016).

[44] A. Banerjee, J. Yan, J. Knolle, C. A. Bridges, M. B. Stone, M. D. Lumsden, D. G. Mandrus, D. A. Tennant, R. Moessner, and S. E. Nagler, Science 356, 1055 (2017).

[45] A. Banerjee, P. Lampen-Kelley, J. Knolle, C. Balz, A. A. Aczel, B. Winn, Y. Liu, D. Pajerowski, J. Yan, C. A. Bridges, A. T. Savici, B. C. Chakoumakos, M. D. Lumsden, D. A. Tennant, R. Moessner, D. G. Mandrus, and S. E. Nagler, npj Quantum Materials 3, 8 (2018).

[46] R. Peierls, Zeitschrift für Physik 80, 763 (1933).

[47] J. Radić, A. Di Ciolo, K. Sun, and V. Galitski, Phys. Rev. Lett. 109, 085303 (2012).

[48] K. Riedl, Y. Li, S. M. Winter, and R. Valentí, Phys. Rev. Lett. 122, 197202 (2019).

[49] H.-S. Kim and H.-Y. Kee, Phys. Rev. B 93, 155143 (2016).

[50] L. Opherden, T. Herrmannsdörfer, M. Uhlarz, D. I. Gorbunov, A. Miyata, O. Portugall, I. Ishii, T. Suzuki, H. Kaneko, H. Suzuki, and J. Wosnitza, Phys. Rev. B 99, 085132 (2019).

[51] I. Rousochatzakis and N. B. Perkins, Phys. Rev. Lett. 118, 147204 (2017).

[52] L.-M. Duan, E. Demler, and M. D. Lukin, Phys. Rev.
Lett. 91, 090402 (2003).

[53] A. Micheli, G. K. Brennen, and P. Zoller, Nature Physics 2, 341 (2006), doi.org/10.1038/nphys287.

[54] D. L. Campbell, G. Juzeliūnas, and I. B. Spielman, Phys. Rev. A 84, 025602 (2011).

[55] M. Lewenstein, A. Sanpera, V. Ahufinger, B. Damski, A. Sen(De), and U. Sen, Advances in Physics 56, 243 (2007), https://doi.org/10.1080/00018730701223200 .

[56] N. Cooper, Advances in Physics 57, 539 (2008), https://doi.org/10.1080/00018730802564122 .

[57] K. A. Modic, T. E. Smidt, I. Kimchi, N. P. Breznay, A. Biffin, S. Choi, R. D. Johnson, R. Coldea, P. WatkinsCurry, G. T. McCandless, J. Y. Chan, F. Gandara, Z. Islam, A. Vishwanath, A. Shekhter, R. D. McDonald, and J. G. Analytis, Nature Communications 5, 4203 (2014).

[58] T. Takayama, A. Kato, R. Dinnebier, J. Nuss, H. Kono, L. S. I. Veiga, G. Fabbris, D. Haskel, and H. Takagi, Physical Review Letters 114, 077202 (2015).

[59] S.-H. Jang, R. Sano, Y. Kato, and Y. Motome, arXiv e-prints , arXiv:1807.01443 (2018), arXiv:1807.01443 [cond-mat.str-el] .

[60] Z.-X. Luo and G. Chen, arXiv e-prints , arXiv:1903.02530 (2019), arXiv:1903.02530 [cond-mat.str-el] .

[61] H. Liu and G. Khaliullin, Phys. Rev. B 97, 014407 (2018).

[62] R. Sano, Y. Kato, and Y. Motome, Phys. Rev. B 97, 014408 (2018). 